\title{
Postoperative outcome of three different procedures for childhood glaucoma
}

This article was published in the following Dove Press journal:

Clinical Ophthalmology

\section{Hailong Huang \\ Wenjun J Bao \\ Tetsuya Yamamoto \\ Kazuhide Kawase \\ Akira Sawada}

Department of Ophthalmology, Gifu University Graduate School of Medicine, Gifu, Japan
Correspondence: Akira Sawada Department of Ophthalmology, Gifu University Graduate School of Medicine, I-I Yanagido, Gifu-shi 50I-I I94, Japan $\mathrm{Tel} / \mathrm{fax}+8 \mathrm{I} 582306288$

Email sawadaa-gif@umin.ac.jp
Purpose: To investigate the long-term postoperative outcome of three surgical procedures for childhood glaucoma.

Patients and methods: In this retrospective study, the patients were divided into a goniotomy group, a trabeculotomy group, and a filtering surgery group, based on the initial surgical procedure. Failure was defined as an IOP $\geq 21 \mathrm{mmHg}$ with medication at two consecutive visits. A Kaplan-Meier analysis was applied to calculate the probability of success. Additional metrics included IOP, number of additional operations, eye drop scores, and visual acuity.

Results: We studied 40 eyes of 25 patients, 21 eyes of 15 patients, and 12 eyes of 7 patients in the goniotomy, trabeculotomy, and filtering surgery groups, respectively. The 10- and 20-year probability of success was $65.2 \%$ and $65.2 \%, 42.2 \%$ and NA (no data for 20 years), and $91.7 \%$ and $80.2 \%$ for the goniotomy, trabeculotomy, and filtering surgery groups, respectively.

Conclusion: All three procedures maintained an IOP of less than $21 \mathrm{mmHg}$ for up to 10 years in $65.2 \%, 42.2 \%$, and $91.7 \%$ of childhood glaucoma cases.

Keywords: childhood glaucoma, goniotomy, trabeculotomy, filtering surgery, long-term outcome

\section{Introduction}

Childhood glaucoma is a disorder characterized by increased IOP due to developmental malformation and associated malfunction of the anterior chamber angle. ${ }^{1}$ Surgical intervention is usually required because correction of the underlying anatomical abnormality is essential. The choice among surgical techniques must be based on associated conditions such as corneal opacity and the type of childhood glaucoma, and the most common procedures are goniotomy and trabeculotomy. Filtering surgery and implantation of a glaucoma drainage device are indicated for selected cases. If the first procedure fails to control IOP, a second procedure is indicated. ${ }^{2}$ We employ goniotomy or trabeculotomy basically as the initial procedure, and filtering surgery in more complicated cases. There are several reports discussing the outcome of goniotomy and trabeculotomy in childhood glaucoma. ${ }^{3-8}$ To the best of our knowledge, only one report exists regarding comparison among these three techniques for childhood glaucoma. ${ }^{9}$ There are some reports using combined trabeculotomy-trabeculectomy surgery, but there are few reports on filtering surgery with mitomycin C (MMC) for childhood glaucoma. ${ }^{10-14}$ Therefore, we analyzed the long-term outcome of childhood glaucoma treated by these three surgical techniques.

\section{Patients and methods Study design}

This is a retrospective study. This study was approved by the ethics committee of Gifu University Graduate School of Medicine (Approval number: 27-306). 
The study was conducted in adherence to the Declaration of Helsinki. All guardians of the patients were required to sign informed consent forms before the performance of any surgical procedures.

\section{Patient selection}

Selection of surgical technique for childhood glaucoma depends on the patient's conditions. Our strategy for the selection is basically as follows: goniotomy or trabeculotomy is primarily indicated according to the surgeon's decision unless visibility of the chamber angle is disturbed. If disturbed, then trabeculotomy is used. Trabeculectomy is indicated when Schlemm's canal cannot be seen during trabeculotomy. Thermal sclerectomy is indicated when a filtering surgery is indicated, but, the case is not old enough to receive postoperative laser suture lysis or other postoperative interventions. Thermal sclerostomy is also indicated for more severe cases such as the ones where the identification of Schlemm's canal is anticipated to be difficult or a severe malformation of the anterior segment is present. Finally, the two filtering surgeries are indicated when additional canal surgery may not be effective.

We retrospectively reviewed the medical records of patients with various types of childhood glaucoma in which goniotomy, trabeculotomy, or filtering surgery was performed as the initial surgery at Gifu University Hospital (Gifu, Japan) between May 1985 and December 2010. The inclusion criteria for the present study were a postoperative follow-up period of more than 2 years and an age of less than 18 years at the initial surgery. Childhood glaucoma cases that did not match the inclusion criteria were excluded. Based on the type of initial surgery, the patients were divided into a goniotomy group, a trabeculotomy group, or a filtering surgery group. Filtering surgery includes trabeculectomy and thermal sclerostomy (Scheie's operation). Before the surgery, we conducted thorough ophthalmic examinations including slit-lamp biomicroscopy, tonometry, measurement of corneal diameter by calipers, gonioscopy, fundus examination, gonioscopy, and visual acuity test and perimetry if indicated. IOP was assessed using a Tono-Pen ${ }^{\circledR}$ Applanation Tonometer (Reichert Technologies, Depew, NY, USA) or an iCare tonometer (Icare Finland Oy, Vantaa, Finland) under general anesthesia in an operating room, and using a Goldmann applanation tonometer, Tono-Pen $\AA$ Applanation Tonometer, or an iCare tonometer in the clinic, in each case.

\section{Surgical procedures}

Goniotomy was performed under general anesthesia. After putting a surgical goniolens such as a Swan-Jacob goniolens on the cornea, a gonioknife (Handaya Co. Ltd, Tokyo, Japan) was inserted into the anterior chamber through the peripheral cornea to the trabecular meshwork on the opposite side. The trabecular meshwork was then incised for approximately $90^{\circ}-120^{\circ}$. After the procedure, the cornea was closed using a 10-0 nylon suture. ${ }^{15}$

Trabeculotomy was conducted under either general or retrobulbar anesthesia according to the methods described elsewhere. ${ }^{16}$ In brief, after conjunctival incision, a $4 \times 4 \mathrm{~mm}$ square or triangular single or double scleral flap was created at the corneal limbus. After identification of Schlemm's canal, its outer wall was cut with a razor blade and excised with fine scissors. U-shaped probes (Nagata trabeculotome probe; Inami \& Co., Ltd, Tokyo, Japan) were then inserted into both ends of the opened Schlemm's canal and rotated $90^{\circ}$ against the trabecular meshwork. Rotation of these probes usually achieved an approximately $120^{\circ}$ opening of the trabecular meshwork. The scleral flap was closed with four 10-0 nylon sutures. A sclerotomy of $1 \mathrm{~mm}$ in diameter, known as a sinusotomy, was created with a punch at one or two locations of the scleral flap before closure of the flap with 10-0 nylon sutures in some cases, according to the method of Mizoguchi et al. ${ }^{17}$

Trabeculectomy was performed under general anesthesia according to the method of Cairns, with some modifications. ${ }^{18}$ Our conjunctival incisions included both limbal-based and fornix-based procedures. After the creation of a scleral flap, sponges soaked with MMC $(0.4 \mathrm{mg} / \mathrm{mL})$ were applied for 5 minutes to the posterior surface of the conjunctiva, Tenon's capsule, the adjacent episcleral tissue, and the scleral flap, followed by copious irrigation with a balanced salt solution. ${ }^{19}$ A trabecular block was excised to create a fistula into the anterior chamber, and peripheral iridectomy was then performed. The scleral flap was closed with 10-0 nylon sutures and the conjunctival flap was also closed with 10-0 nylon or 10-0 absorbable sutures.

We performed a thermal sclerostomy, sometimes described as a peripheral iridectomy with scleral cauterization, according to the method of Scheie. ${ }^{20}$ A conjunctival incision was made in a limbal-based fashion. After partially incising the limbal tissue, sponges soaked with MMC were applied as described above for trabeculectomy. ${ }^{19}$ Following the thermal cautery at the partially incised limbal tissue, a perforating incision to the anterior chamber was made, which was followed by peripheral iridectomy. The conjunctival flap was closed with 10-0 nylon or 10-0 absorbable sutures.

\section{Main outcome measure}

The main outcome measure was the probability of success of surgery based on a Kaplan-Meier analysis. We did not 
regard repeated operations with the same surgical technique as a failure in this analysis because the aim of this study is to investigate the final success probability of each surgical technique. Surgical failure was defined as one of the following three: 1) when additional glaucoma filtering surgery was performed; 2) visual acuity deteriorated to an absence of light perception; and 3) an IOP greater than or equal to $21 \mathrm{mmHg}$ with ocular hypotensive medications, verified during two consecutive visits. The date at which the first such IOP was observed was recorded as the date of the failure. IOPs measured within 3 months after a surgery were not considered a surgical failure to account for normal early postoperative IOP fluctuations, especially after trabeculotomy. As for failure criteria 1 and 2, the eye was regarded as a surgical failure at that time point. In addition, in the filtering surgery group only, we focused on two types of surgical outcomes, defining a need for additional filtering surgeries as a failure in one and not in the other.

\section{Statistical analysis}

Data analysis was performed using Bell Curve for Excel ver. 2.00 (Social Survey Research Information Co., Ltd, Tokyo Japan). The probability of success was subjected to a log-rank test after a Kaplan-Meier analysis. In addition, the IOP was compared before and after surgery using a Wilcoxon signedrank test. The number of additional surgeries, eye drop scores, final visual acuity, and final IOP were compared using the Steel-Dwass method. $P$-value of $<0.05$ was considered to be statistically significant.

\section{Results}

A total of 73 eyes of 43 patients were analyzed in the present study. The type of childhood glaucoma was primary congenital glaucoma in 49 eyes, juvenile open-angle glaucoma in 10 eyes, and secondary childhood glaucoma in 14 eyes. The age at the initial surgery was $4.1 \pm 6.4$ years (mean $\pm \mathrm{SD}$; range: 1 month to 17.7 years). The follow-up period was 9.6 \pm 6.7 years (range: 2-20 years). Forty eyes of 25 patients, 21 eyes of 15 patients, and 12 eyes of 7 patients underwent initial surgery with goniotomy, trabeculotomy, and filtering surgery, respectively. Filtering surgery included trabeculectomy in 8 eyes of 6 patients and thermal sclerostomy in 4 eyes of 3 patients. The preoperative IOP was $26.8 \pm 9.4 \mathrm{mmHg}$ (range: $12-58 \mathrm{mmHg}$ ) and the final IOP was $14.8 \pm 5.3 \mathrm{mmHg}$ (range: $4-30 \mathrm{mmHg}$ ) for all cases. The final IOP was significantly decreased in all groups when compared with the preoperative value (Wilcoxon signed-rank test). The age at surgery was significantly lower in the goniotomy group compared with the other two groups and the follow-up period was significantly shorter in the trabeculotomy group compared with the other two groups $(P=0.025$ and $P=0.001$, respectively; Wilcoxon signed-rank test) (Table 1).

The number of additional surgeries required is shown in Figure 1. In the goniotomy group, 15 eyes (38\%) received a second goniotomy and 9 eyes $(60 \%)$ received a third surgery. In the trabeculotomy group, a second trabeculotomy or filtering surgery was performed in 17 eyes (80.9\%), followed by the third surgery in 11 eyes (65\%). In the filtering surgery group, a second filtration surgery was performed in 6 eyes $(50.0 \%)$, followed by a third such surgery in 2 eyes $(33 \%)$ (Figure 1).

Figure 2 presents the probability of success calculated using a Kaplan-Meier analysis. The probability of success was $65.2 \% \pm 7.8 \%$ and $65.2 \% \pm 7.8 \%$ (estimated probability \pm standard error) at 10 and 20 years after the initial surgery, respectively, in the goniotomy group. It was $42.2 \% \pm 13.6 \%$ at 10 years in the trabeculotomy group. Similarly, it was $91.7 \% \pm 8.0 \%$ and $80.2 \% \pm 12.8 \%$ at 10 and 20 years after the initial surgery in the filtering surgery group. Only the filtering surgery group showed significantly higher probability of success than did the trabeculotomy group at 10 years $(P=0.030$ : trabeculotomy vs filtering

Table I Patients' characteristics

\begin{tabular}{l|l|l|l|l}
\hline Group & Goniotomy & Trabeculotomy & Filtering surgery & All \\
\hline Number of patients (eyes) & 25 patients & 15 patients & 7 patients & 43 patients \\
& $(40$ eyes $)$ & $(21$ eyes $)$ & $(12$ eyes) & $(73$ eyes $)$ \\
$28 / 15$ & $4 / 3$ & $49 / 10 / 14$ \\
Sex (male/female) & $18 / 7$ & $8 / 7$ & $7 / 2 / 3$ & \\
Type of childhood glaucoma (primary congenital/ & $34 / 0 / 6$ & $8 / 8 / 5$ & & \\
juvenile open angle/secondary childhood) (eyes) & & & \\
Age at surgery (years) & $1.3 \pm 1.9$ & $5.6 \pm 6.8$ & $6.9 \pm 8.0$ & $9.1 \pm 6.4$ \\
Follow-up period (years) & $13.3 \pm 5.8$ & $6.3 \pm 2.8$ & $13.4 \pm 6.6$ & $9.6 \pm 6.7$ \\
Preoperative IOP (mmHg) & $28.0 \pm 8.5$ & $25.5 \pm 10.2$ & $25.4 \pm 9.2$ & $26.8 \pm 9.4$ \\
Final IOP (mmHg) & $13.8 \pm 4.3$ & $17.7 \pm 4.1$ & $13.4 \pm 6.6$ & $14.8 \pm 5.3$ \\
P-value* & $<0.001$ & 0.009 & 0.016 & $<0.001$ \\
\hline
\end{tabular}

Note: *Between preoperative and final IOPs (Wilcoxon signed-rank test). 


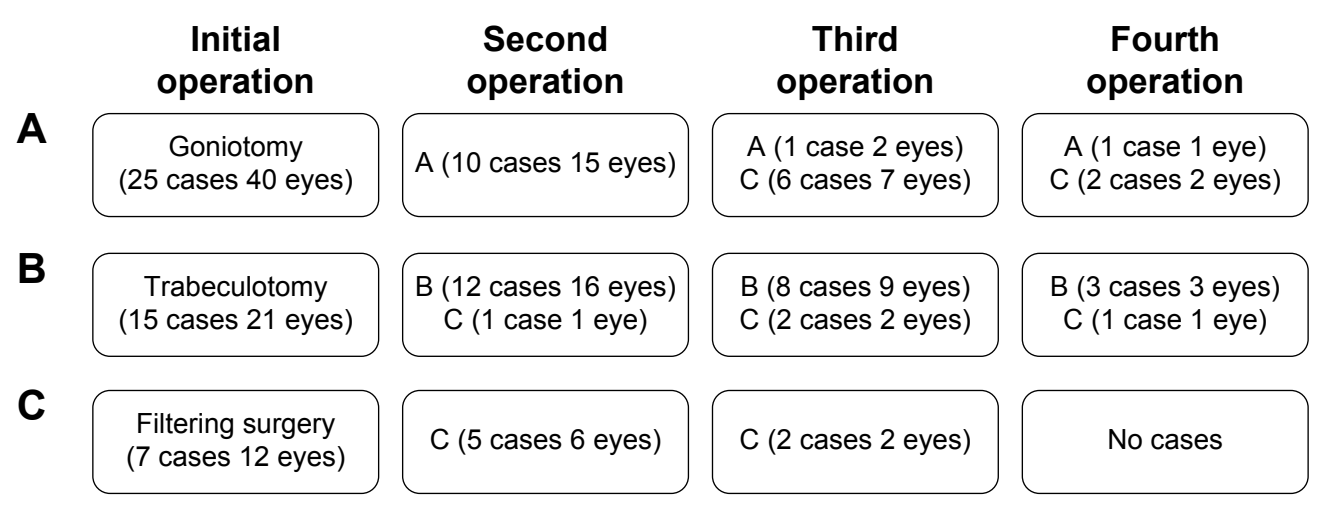

Figure I Additional surgeries.

Notes: (A) Goniotomy; (B) trabeculotomy; and (C) filtering surgery. In the goniotomy group, 15/40 eyes (38\%) received a second goniotomy, followed by additional surgery in $7 / 15$ eyes (47\%). In the trabeculotomy group, a second trabeculotomy or filtering surgery was performed on I7/2I eyes (8I\%), followed by a third additional surgery in $9 / 17$ (53\%). In the filtering surgery group, $6 / 12$ eyes (50\%) underwent a second filtration surgery, followed by additional surgeries in $2 / 6$ eyes ( $33 \%$ ).

surgery, $P=0.157$ : goniotomy vs trabeculotomy, $P=0.273$ : goniotomy vs filtering surgery; Cochran-Mantel-Haenszel test). Where we considered the need for an additional filtering surgery as a failure, the probability of success was reduced to $58.3 \% \pm 14.2 \%$ and $35.0 \% \pm 15.4 \%$ at 10 and 20 years, respectively (Figure 3 ).

The trabeculotomy group needed significantly more additional surgeries than the other two groups $(P=0.048$ : trabeculotomy vs filtering surgery, $P=0.049$ : goniotomy vs trabeculotomy, $P=0.856$ : goniotomy vs filtering surgery; Steel-Dwass test) (Figure 4A). The final medication number was significantly higher in the trabeculotomy group than in the other two groups $(P<0.001$ : trabeculotomy vs filtering surgery, $P<0.001$ : goniotomy vs trabeculotomy, $P=0.954$ : goniotomy vs filtering surgery; Steel-Dwass test) (Figure 4B). The final visual acuity was significantly lower in the filtration surgery group than in the other two groups ( $P=0.006$ : trabeculotomy vs filtering surgery, $P=0.997$ :

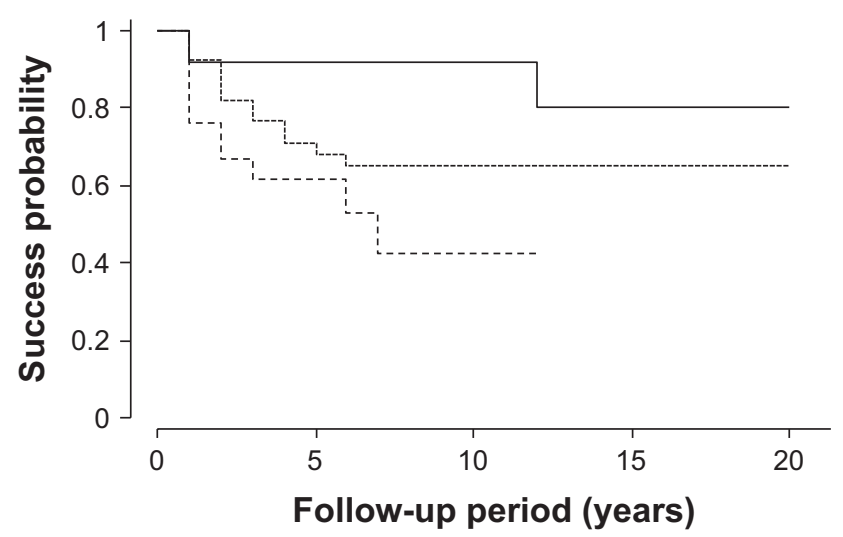

Figure 2 Probability of success.

Note: Dotted line: goniotomy group; broken line: trabeculotomy group; and solid line: filtering surgery group. goniotomy vs trabeculotomy, $P=0.011$ : goniotomy vs filtering surgery; Steel-Dwass test) (Figure 4C). Figure 4D shows the final IOPs.

\section{Discussion}

Childhood glaucoma is a recalcitrant glaucoma subtype because medical therapy is often ineffective and pathological change is more obvious and severe in the outflow channel than in primary glaucoma. Trabecular surgery, and primarily goniotomy or trabeculotomy, is indicated in these cases. In more severe cases, such as in instances of severe corneal opacity or deformed anterior segment, glaucoma drainage devices or filtering surgery is indicated. In the present retrospective study with a mean follow-up of 9.6 years, we found that the probability of success of goniotomy and trabeculotomy was $65.2 \%$ and $42.2 \%$ at 10 -year follow-up, respectively. For filtering surgery, including trabeculectomy and thermal sclerostomy, it was $91.7 \%$. The indications

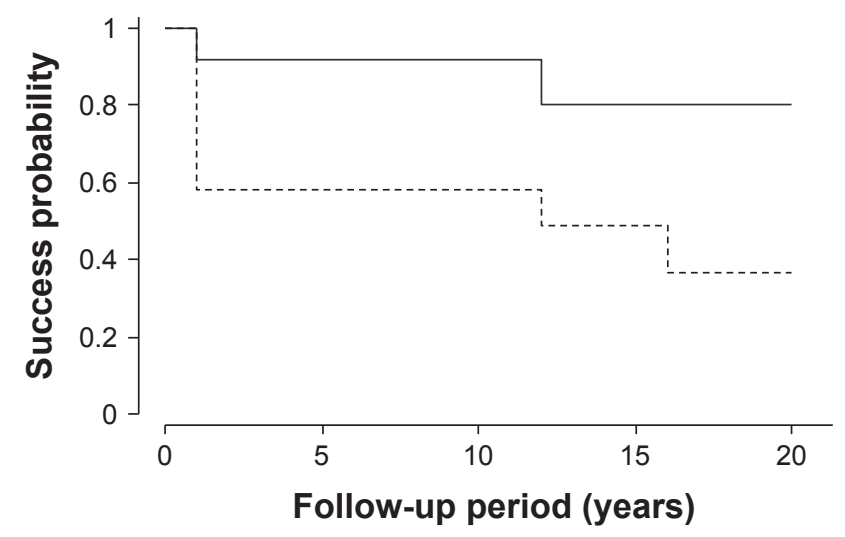

Figure 3 Probability of success in the filtering surgery group.

Note: Broken line: where conducting additional filtering surgeries was regarded as a failure; and solid line: where it was not regarded as a failure. 
A
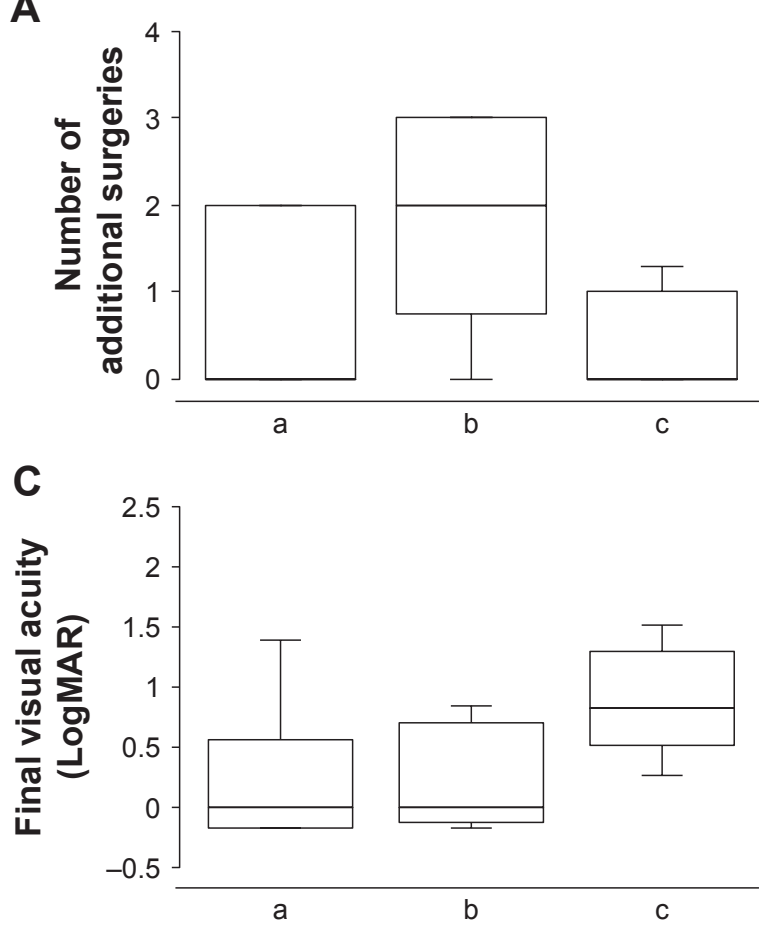

B

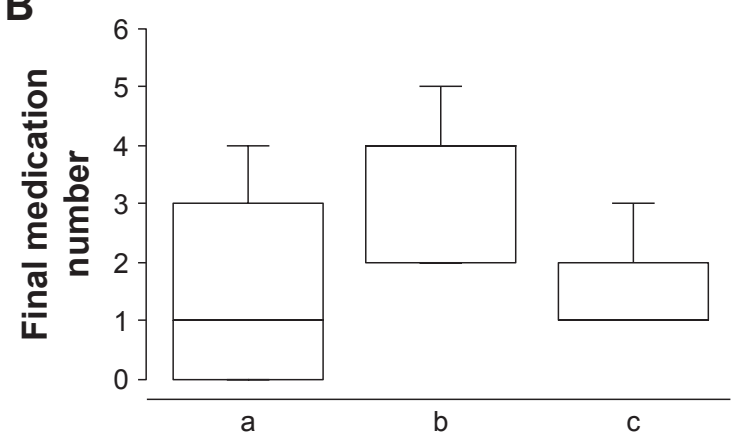

D

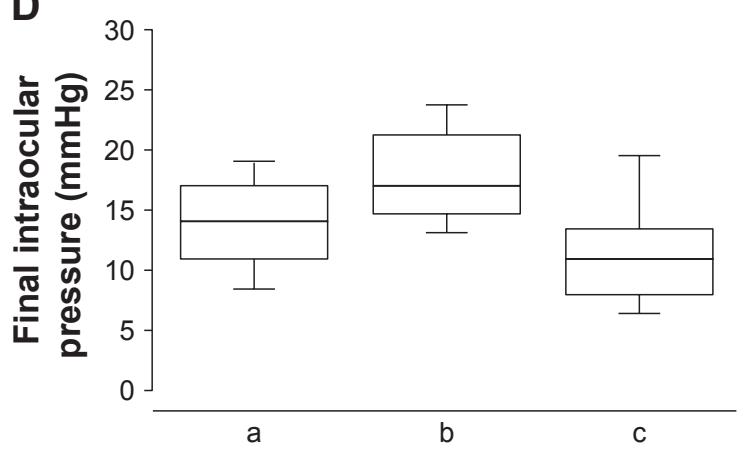

Figure 4 Postoperative clinical data.

Notes: (A) Number of additional surgeries; (B) final number of glaucoma medications in use; (C) final visual acuity (LogMAR); and (D) final intraocular pressure. a: Goniotomy grou; b: trabeculotomy group; and c: filtering surgery group. The box and whisker plot shows the range and the first, second, and third quartiles of the values.

for each of the three surgical procedures are, of course, quite different. As mentioned above, our basic strategy is as follows: in cases with childhood glaucoma, goniotomy or trabeculotomy is indicated according to the surgeon's decision unless visibility of the chamber angle is disturbed. Trabeculectomy is indicated when Schlemm's canal cannot be seen during trabeculotomy. Thermal sclerectomy is indicated for cases that are not old enough to receive postoperative laser suture lysis or other postoperative intervention or more severe cases such as a severe malformation of the anterior segment is present. Finally, the two filtering surgeries are indicated when additional canal surgery may not be effective.

Goniotomy was first reported in 1948 by Otto Barkan. ${ }^{21}$ Shaffer ${ }^{22}$ investigated and reported the surgical success rate of this procedure, defining success as an IOP of less than or equal to $20 \mathrm{mmHg}$ over a 6-month follow-up. He reported success rates of $94 \%$ in cases where surgery was done between the ages of 1 month and 2 years, $26 \%$ in cases done at age less than 1 month, and $38 \%$ in cases done at greater than 2 years. ${ }^{22}$ The 5 - and 10-year success rates, defined as an IOP of less than or equal to $20 \mathrm{mmHg}$, were $61.4 \%$ at both time points in our previous study, ${ }^{23}$ which included some of the same patients analyzed in the present study. The present study found a success rate of $65.2 \%$ at both 10 and 20 years postoperatively.
Thus, long-term stability of IOP can be expected following goniotomy. We also found that goniotomy cases needed fewer additional surgeries and fewer medications and that the final IOP was lower than in the trabeculotomy cases. The better outcome with goniotomy probably reflects the milder clinical presentation in these cases, such as a clearer cornea. However, Zagora et $\mathrm{al}^{9}$ recently reviewed their medical records with primary congenital glaucoma over a 23-year period. They reported that success rate, which was defined as an IOP of less than or equal to $21 \mathrm{mmHg}$, was only $26.4 \%$ with up to two goniotomies. ${ }^{9}$

The advantage of trabeculotomy is that corneal opacity, a common sign of childhood glaucoma, does not affect the procedure. Thus, the indication for trabeculotomy is broader than that for goniotomy. Luntz reported a trabeculotomy success rate of $89.5 \%$ at 6.5 years postoperatively in a series of 86 cases at ages $2-12$ years. ${ }^{24}$ Zagora et al also found a high success rate of $78.95 \%$ for primary trabeculotomy, which was defined as an IOP of less than or equal to $21 \mathrm{mmHg}^{9}$ A Japanese study reported success rates of $94.3 \%, 87.7 \%$, and $80.8 \%$ at 5,10 , and 20 years, respectively, defining successful IOP as less than $21 \mathrm{mmHg}$ and no need for additional surgery. ${ }^{25}$ Other Japanese group reported success rates of $93.0 \%, 85.0 \%$, and $68.0 \%$ at 100,200 , and 300 months up to 
three trabeculotomies, respectively, defining successful IOP as less than $21 \mathrm{mmHg} .{ }^{26}$ Yassin and Al-Tamimi found success rates of around $80.0 \%$ and $60.0 \%$ at 5 and 10 years against the number of trabeculotomies performed, respectively, defining successful IOP as less than $21 \mathrm{mmHg} .{ }^{27}$ As stated above, the present study found trabeculotomy to be inferior to other procedures in terms of IOP control and need of medication, although, as noted, this may simply reflect the more severe condition of the eyes recruited into the trabeculotomy group. There are several reports of trabeculotomy combined with trabeculectomy in children. ${ }^{28-30}$ The results are inconsistent, but some of them are promising. ${ }^{28}$

A majority of ophthalmologists believe that filtering surgery is not a primary surgical procedure for childhood glaucoma. However, it is indicated in some cases, such as those in which the outflow channel is quite deformed and anatomical recovery via canal surgery is unlikely. It should be noted that adjunctive use of antimetabolites such as MMC or 5-fluorouracil (5-FU) is usually indicated in children due to their more robust healing responses that can cause massive scar formation. ${ }^{31}$ There are several reports of trabeculectomy in childhood glaucoma. Freedman et al reported a success rate of $52.4 \%$ in 21 eyes that had undergone trabeculectomy with 5-FU and that the success rate was age-dependent: $73.0 \%$ and $30.0 \%$ for cases older than 1 year and younger than 1 year, respectively. ${ }^{32}$ Dureau et al reported a final success rate of $87.3 \%$, noting that $31 \%$ of the cases needed repeated trabeculectomies. ${ }^{33}$ Fulcher et al reported that $92.3 \%$ of cases achieved control in primary infantile glaucoma after a single trabeculectomy and that $85.7 \%$ did so in secondary infantile glaucoma. ${ }^{34}$ Yassin and Al-Tamimi found success rates of around 70.0\% and $65.0 \%$ at 5 and 10 years against the number of trabeculectomies performed, respectively, defining successful IOP as less than $21 \mathrm{mmHg} .{ }^{27}$ The present success rates of $91.7 \%$ and $80.2 \%$ after 10 and 20 years, respectively, in the filtering surgery group, are consistent with these previous reports. The good results with filtering surgery in the present study may reflect the use of thermal sclerostomy in many cases, which reduced the need of postoperative IOP modulation via laser suture lysis or needling, a difficult procedure in children.

The present study has several limitations. First, it was a retrospective study. Second, the indications for the surgeries were varied, which could introduce selection bias among the treatment groups. Third, the number of patients was relatively small. However, we did include long follow-up periods that greatly enhance the clinical relevance of this study.

\section{Conclusion}

The present retrospective study found that the probability of success, defined as maintenance of an IOP of less than 21 $\mathrm{mmHg}$ irrespective of ocular hypotensive medications, was $65.2 \%, 42.2 \%$, and $91.7 \%$ in the goniotomy group, trabeculotomy group, and filtering surgery group, respectively, at 10 years postoperatively. These three surgical procedures provide valuable options for the treatment of childhood glaucoma, a historically recalcitrant disease.

\section{Disclosure}

KK reports lecture fees from Kowa (Nagoya, Japan) and Santen (Osaka, Japan). TY reports grants from Alcon Japan (Tokyo, Japan), Alcon Pharma (Tokyo, Japan), MSD Japan (Tokyo, Japan), Otsuka (Tokyo, Japan), Pfizer Japan (Tokyo, Japan), Santen (Osaka, Japan), and Senju (Osaka, Japan); consultant/advisor fees from Alcon Japan (Tokyo, Japan), Kowa (Nagoya, Japan), Rohto (Osaka, Japan), Otsuka (Tokyo, Japan), Pfizer Japan (Tokyo, Japan), and Senju (Osaka, Japan); and lecture fees from Johnson \& Johnson (Tokyo, Japan), Novartis Pharma (Tokyo, Japan), R-Tech Ueno (Tokyo, Japan), AMO Japan (Tokyo, Japan), CREWT Medical Systems (Tokyo, Japan), Alcon Japan (Tokyo, Japan), Kowa (Nagoya, Japan), MSD Japan (Tokyo, Japan), Otsuka (Tokyo, Japan), Pfizer Japan (Tokyo, Japan), Santen (Osaka, Japan), and Senju (Osaka, Japan). AS reports lecture fees from Alcon Japan (Tokyo, Japan), Kowa (Nagoya, Japan), Pfizer Japan (Tokyo, Japan), Santen (Osaka, Japan), and Senju (Osaka, Japan). The authors report no other conflicts of interest in this study.

\section{References}

1. Dickens CJ, Hoskins HD. Epidemiology and pathology of congenital glaucoma. In: Ritch R, Shields MB, Krupin T, editors. The Glaucomas Vol II Clinical Science. St Louis: CV Mosby; 1996:729-738.

2. Chen TC, Chen PP, Francis BA, et al. Pediatric glaucoma surgery: a report by the American Academy Of Ophthalmology. Ophthalmology. 2014; 121(11):2107-2115.

3. McPherson SD Jr, McFarland D. External trabeculotomy for developmental glaucoma. Ophthalmology. 1980;87(4):302-305.

4. McPherson SD Jr, Berry DP. Goniotomy vs external trabeculotomy for developmental glaucoma. Am J Ophthalmol. 1983;95(4):427-431.

5. Gramer E, Tausch M, Kraemer C. Time of diagnosis, reoperations and long-term results of goniotomy in the treatment of primary congenital glaucoma: a clinical study. Int Ophthalmol. 1996-1997;20(1-3):117-123.

6. Anderson DR. Trabeculotomy compared to goniotomy for glaucoma in children. Ophthalmology. 1983;90(7):805-806.

7. Meyer G, Schwenn O, Pfeiffer N, Grehn F. Trabeculotomy in congenital glaucoma. Graefes Arch Clin Exp Ophthalmol. 2000;238(3):207-213.

8. Mendicino ME, Lynch MG, Drack A, et al. Long-term surgical and visual outcomes in primary congenital glaucoma: 360 degrees trabeculotomy versus goniotomy. J AAPOS. 2000;4(4):205-210. 
9. Zagora SL, Funnell CL, Martin FJ, et al. Primary congenital glaucoma outcomes: lessons from 23 years of follow-up. Am J Ophthalmol. 2015; 159(4):788-796

10. Elder MJ. Combined trabeculotomy-trabeculectomy compared with primary trabeculectomy for congenital glaucoma. Br J Ophthalmol. 1994;78(10):745-748.

11. Mullaney PB, Selleck C, Al-Awad A, Al-Mesfer S, Zwaan J. Combined trabeculotomy and trabeculectomy as an initial procedure in uncomplicated congenital glaucoma. Arch Ophthalmol. 1999;117(4):457-460.

12. Dietlein TS, Jacobi PC, Krieglstein GK. Prognosis of primary ab externo surgery for primary congenital glaucoma. Br J Ophthalmol. 1999;83(3):317-322.

13. Mandal AK, Bhatia PG, Bhaskar A, Nutheti R. Long-term surgical and visual outcomes in Indian children with developmental glaucoma operated on within 6 months of birth. Ophthalmology. 2004;111(2):283-290.

14. Lawrence SD, Netland PA. Trabeculectomy versus combined trabeculotomy-trabeculectomy in pediatric glaucoma. J Pediatr Ophthalmol Strabismus. 2012;49(6):359-365.

15. Hoskins HD Jr, Shaffer RN, Hetherington J. Goniotomy vs trabeculotomy. J Pediatr Ophthalmol Strabismus. 1984;21(4):153-158.

16. Tanihara H, Negi A, Akimoto M, et al. Surgical effects of trabeculotomy ab externo on adult eyes with primary open angle glaucoma and pseudoexfoliation syndrome. Arch Ophthalmol. 1993;111(12):1653-1661.

17. Mizoguchi T, Nagata M, Matsumura M, Kuroda S, Terauchi H, Tanihara H. Surgical effects of combined trabeculotomy and sinusotomy compared to trabeculotomy alone. Acta Ophthalmol Scand. 2000;78(2):191-195.

18. Cairns JE. Trabeculectomy. Preliminary report of a new method. Am J Ophthalmol. 1968;66(4):673-679.

19. Kitazawa Y, Suemori-Matsushita H, Yamamoto T, Kawase K. Low-dose and high-dose mitomycin trabeculectomy as an initial surgery in primary open-angle glaucoma. Ophthalmology. 1993;100(11):1624-1628.

20. Scheie HG. Retraction of scleral wound edges; as a fistulizing procedure for glaucoma. Am J Ophthalmol. 1958;45(4 Pt 2):220-229.

21. Barkan O. Goniotomy for the relief of congenital glaucoma. $\mathrm{Br} J$ Ophthalmol. 1948;32(9):701-728.

22. Shaffer RN. Prognosis of goniotomy in primary infantile glaucoma (trabeculodysgenesis). Trans Am Ophthalmol Soc. 1982;80:321-325.
23. Kondo N, Sawada A, Kawase K, Yamamoto T, Kitazawa Y. Outcome of goniotomy for congenital glaucoma. Atarashii Ganka. 2003;20(7): 981-984.

24. Luntz MH. Congenital, infantile, and juvenile glaucoma. Ophthalmology. 1979;86(5):793-802.

25. Ikeda H, Ishigooka H, Muto T, Tanihara H, Nagata M. Long-term outcome of trabeculotomy for the treatment of developmental glaucoma. Arch Ophthalmol. 2004;122(8):1122-1128.

26. Ozawa H, Yamane M, Inoue E, et al. Long-term surgical outcome of conventional trabeculotomy for childhood glaucoma. Jpn JOphthalmol. 2017;61(3):237-244.

27. Yassin SA, Al-Tamimi ER. Surgical outcomes in children with primary congenital glaucoma: a 20-year experience. Eur J Ophthalmol. 2016 26(6):581-587.

28. Singab AAS, Mohammed OA, Saleem MIH, Abozaid MA. A comparative study: the use of collagen implant versus mitomycin-C in combined trabeculotomy and trabeculectomy for treatment of primary congenital glaucoma. J Ophthalmol. 2017;2017:9241459.

29. Khalil DH, Abdelhakim MA. Primary trabeculotomy compared to combined trabeculectomy-trabeculotomy in congenital glaucoma: 3-year study. Acta Ophthalmol. 2016;94(7):e550-e554.

30. Temkar S, Gupta S, Sihota R, et al. Illuminated microcatheter circumferential trabeculotomy versus combined trabeculotomy-trabeculectomy for primary congenital glaucoma: a randomized controlled trial. Am J Ophthalmol. 2015;159(3):490-497.e2.

31. Snir M, Lusky M, Shalev B, Gaton D, Weinberger D. Mitomycin C and 5-fluorouracil antimetabolite therapy for pediatric glaucoma filtration surgery. Ophthalmic Surg Lasers. 2000;31(1):31-37.

32. Freedman SF, McCormick K, Cox TA. Mitomycin C-augumented trabeculectomy with postoperative wound modulation in pediatric glaucoma. J AAPOS. 1999;3(2):117-124.

33. Dureau P, Dollfus H, Cassegrain C, Dufier JL. Long-term results of trabeculectomy for congenital glaucoma. J Pediatr Ophthalmol Strabismus. 1998;35(4):198-202.

34. Fulcher T, Chan J, Lanigan B, Bowell R, O'Keefe M. Long-term follow up of primary trabeculectomy for infantile glaucoma. Br J Ophthalmol. 1996;80(6):499-502.
Clinical Ophthalmology

\section{Publish your work in this journal}

Clinical Ophthalmology is an international, peer-reviewed journal covering all subspecialties within ophthalmology. Key topics include: Optometry; Visual science; Pharmacology and drug therapy in eye diseases; Basic Sciences; Primary and Secondary eye care; Patient Safety and Quality of Care Improvements. This journal is indexed on Submit your manuscript here: http://www.dovepress.com/clinical-ophthalmology-journa

\section{Dovepress}

PubMed Central and CAS, and is the official journal of The Society of Clinical Ophthalmology (SCO). The manuscript management system is completely online and includes a very quick and fair peer-review system, which is all easy to use. Visit http://www.dovepress.com/ testimonials.php to read real quotes from published authors. 